\title{
Hormonal contraceptive use is associated with neural and affective changes in healthy young woments
}

\author{
Nina Lisofsky a,b,*, Michaela Riediger ${ }^{\mathrm{a}}$, Jürgen Gallinat ${ }^{\mathrm{b}}$, Ulman Lindenberger ${ }^{\mathrm{a}, \mathrm{c}}$, Simone Kühn ${ }^{\mathrm{a}, \mathrm{b}}$ \\ a Center for Lifespan Psychology, Max Planck Institute for Human Development, Berlin, Germany \\ ${ }^{\mathrm{b}}$ Department of Psychiatry and Psychotherapy, University Medical Center Hamburg-Eppendorf, Germany \\ c European University Institute, San Domenico di Fiesole, Italy
}

\section{A R T I C L E I N F O}

Article history:

Received 16 December 2015

Revised 13 April 2016

Accepted 18 April 2016

Available online 19 April 2016

\section{Keywords:}

Hormonal contraceptives

Amygdala

Affect

SMRI

Emotion regulation

Functional connectivity

\begin{abstract}
A B S T R A C T
Previous neuroimaging research has demonstrated that female gonadal hormones can alter the structure and function of adult women's brains. So far, we do not know how hormonal contraceptives affect female brain structure, in part because within-person longitudinal observations are lacking. Here, we compared 28 young women before and after three months of regular contraceptive intake with 28 naturally cycling women of comparable age. The goal was to explore within-person neural change in women using contraceptives. Neuroimaging, hormonal, cognitive, and affect data were collected at two time points for each participant. A voxel-wise wholebrain comparison of both groups revealed decreased gray matter volume in the left amygdala/anterior parahippocampal gyrus in women using contraceptives as compared to the control group. Resting-state functional connectivity of this region with the dorsolateral prefrontal cortex changed from positive to negative connectivity following contraceptive intake whereas the opposite held for the control group. An exploratory analysis revealed that gray matter volume in the left amygdala/anterior parahippocampal gyrus was associated with positive affect at the second time point. There were no systematic differences in cognitive performance change between the groups. These findings provide initial insights into effects of hormonal contraceptives on the human brain and expand previous findings on hormone-related amygdala/hippocampal complex plasticity. The affected brain regions may be related to psychological wellbeing, underlining the importance of future studies on contraceptive-induced brain changes.
\end{abstract}

(c) 2016 Elsevier Inc. All rights reserved.

\section{Introduction}

Hormonal contraceptives are the most common contraceptive method in the Western world (Johnson et al., 2013; United Nations, 2015). To give one example, about one third of adult women in Germany use hormonal contraceptive pills (hereafter also "the pill"), with higher proportions found at younger ages (Federal Centre for Health Education (BZgA), 2011; in a sample in the US, 17\% of women aged 15-44 years used the pill, with higher proportions at younger ages: Jones et al., 2012). During the natural menstrual cycle, female gonadal hormones (estrogen and progesterone) change in a regular temporal pattern enabling fertility. Hormonal contraceptives suppress ovulation by modifying the natural fluctuation of gonadal hormones. Given that a growing body of literature indicates that female gonadal hormones have the potential to alter the human adult brain (Comasco

\footnotetext{
is Nina Lisofsky was a pre-doctoral fellow of the International Max Planck Research School on the Life Course (LIFE, www.imprs-life.mpg.de; participating institutions: Max Planck Insitute for Human Development, Freie Universität Berlin, Humboldt-Universität zu Berlin, University of Michigan, University of Virginia, University of Zurich).

* Corresponding author at: MPI for Human Development, Lentzeallee 94, 14195 Berlin, Germany.

E-mail address: Lisofsky@mpib-berlin.mpg.de (N. Lisofsky).
}

et al., 2014; De Bondt et al., 2013; Lisofsky et al., 2015a; Toffoletto et al., 2014), the question arises whether hormonal contraceptives might also change the brain.

Despite a growing literature on effects of hormones on brain plasticity, we know surprisingly little about the ways in which hormonal contraceptives affect their users' brain structure. To our knowledge, there are no longitudinal studies investigating structural brain changes in women following intake of the pill. Some cross-sectional studies compared gray matter volume of women using hormonal contraceptives with that of non-users, yielding inconsistent results (De Bondt et al., 2013; Petersen et al., 2015; Pletzer et al., 2010; Pletzer et al., 2015). In light of potential pre-existing differences between contraceptive-users and non-users, it is not known whether findings obtained in studies with a between-person design will parallel observations based on within-person changes following use of hormonal contraceptives (Oinonen et al., 2008; Raz et al., 2005). Moreover, the studies that have been conducted so far reported effects of contraceptives after relatively long and habitual exposure to contraceptives (mostly over years). Data on more immediate within-person neural changes are lacking.

To the best of our knowledge, only two neuroimaging studies have assessed women before and after exposure to hormonal contraceptives, 
albeit with a focus on functional rather than on structural brain alterations. Both studies used a randomized placebo-controlled design to test differences in brain activity before and after 21 days of hormonal contraceptive intake (Gingnell et al., 2013, 2015). One of the studies applied a cognitive control paradigm and revealed that contraceptive intake had only little effect on brain reactivity during response inhibition (Gingnell et al., 2015). The other study used an emotional face matching task and observed reduced activity in insular and frontal regions in the contraceptive group and reduced activity in the amygdala in the placebo group following treatment (Gingnell et al., 2013). The latter study only included women who had reported previous negative mood effects following hormonal contraceptive use, which limits the generalizability of the results. Nevertheless, the findings add to research showing altered amygdala responses in naturally cycling women after a single progesterone dose (Van Wingen et al., 2008). Altered amygdala reactivity potentially underlies mood changes that have been discussed as relatively common side-effects of hormonal contraception leading to its discontinuation (Rosenberg and Waugh, 1998).

Longitudinal and cross-sectional studies assessing contraceptionrelated mood changes have yielded inconsistent results. The use of hormonal contraceptives was found to be associated with increases, decreases or no overall change in negative affect (Gingnell et al., 2013; Jarva and Oinonen, 2007; O'Connell et al., 2007; Oinonen and Mazmanian, 2002; Ott et al., 2008; Rosenberg and Waugh, 1998; Shahnazi et al., 2014; Svendal et al., 2012). It is unclear whether the inconsistency of findings reflects actual differences between women in their mood-related responses to hormonal contraceptives, or whether they are due to differences in study design or pill types (Pletzer and Kerschbaum, 2014). Potential associations of mood changes and brain structural changes induced by hormonal contraceptives have not yet been investigated.

We conducted a longitudinal magnetic resonance imaging (MRI) study including an age-matched control group with the aim to investigate (1) structural brain alterations following three months of hormonal contraceptive intake in healthy young women, (2) brain function (i.e., resting-state functional connectivity) of regions showing structural alterations in women using hormonal contraceptives, (3) affective changes in women starting to use contraceptives and potential associations with structural brain changes, and (4) the impact of hormonal contraception on cognitive performance. This study part followed up on previous inconsistent findings regarding cognitive functioning in relation to hormonal contraception (Gogos et al., 2014; Warren et al., 2014).

This study focused on the group-by-time interaction in gray matter volume in a whole brain analysis. In previous work, amygdala function was found to be especially sensitive to endogenous as well as exogenous hormonal variation in women (Lisofsky et al., 2015b; Petersen and Cahill, 2015; Van Wingen et al., 2008). Also, altered amygdala activity and amygdala volume were found to correlate in clinical populations (e.g., Kalmar et al., 2009; Siegle et al., 2003). Hence, we hypothesized that amygdala volume and potentially functional connectivity would perhaps also be altered in women using hormonal contraceptives. Based on previous indications of affective changes in women taking contraceptives (e.g., Gingnell et al., 2013), we speculated that three months of contraceptive use might also have an impact on positive and/or negative affect.

\section{Materials and methods}

\subsection{Participants}

Women planning to start hormonal contraceptive (the pill) use were recruited via flyers in local gynecologists' practices. Healthy women aged 16 to 35 were included in the study. Exclusion criteria were: The use of hormonal contraceptives within a period of six months prior to the study phase, previous pregnancy beyond 8 weeks of gestation, hormonal disorders, MR incompatibility, or a history of psychiatric or neurological illness (based on self-report). For participants under the age of 18, written informed consent was obtained from their parents. The study was conducted according to the Declaration of Helsinki, with approval from the Ethics Committee of the German Society for Psychology. Thirty-one women were included in the "pill group." Three participants dropped out before the second measurement time point (one women could not be recontacted and two women did not have time to take part again). Therefore, the final sample consisted of 28 participants in the pill group (mean age 21.25 years, range 16-33 years. Twenty-eight young women of similar age who were not taking hormonal contraceptives were enrolled as a control group (mean age 21.5 years, range $16-28$ years). The same exclusion criteria applied to the participants in the control group. Sample characteristics for both groups are shown in Table 1 .

\subsection{Overview of design and procedure}

The study design is depicted in Fig. 1. All women participated in two measurement occasions: time point 1 (T1) and time point 2 (T2). The timing of test sessions in relation to the participants' menstrual cycle is described in detail below. For participants in the pill group, T1 took place before they started use of hormonal contraceptives. T2 was scheduled in the pill-free week of the third pill cycle (each pill cycle consisted of three weeks continuous daily pill use and one pill-free week, lasting about one month). The rationale for scheduling the second measurement occasion in the pill-free week was to ensure that potential differences in brain structure are not solely driven by acute effects of the last pill intake. For participants in the control group, T2 was scheduled approximately three months after $\mathrm{T} 1$. At each time point, cognitive, psychological, and neuroimaging data were acquired in two test sessions. In the cognitive test session, participants carried out a number of computer-based cognitive tasks (see below) and completed the Positive and Negative Affect Schedule (PANAS; Watson et al., 1988). Throughout the cognitive test session, three saliva samples were provided by the participants. Measurement sessions took place in the morning (starting before noon between 8:00 and 12:00 am), unless this was impossible to schedule for the participants. In the MRI session, participants were measured in the scanner and completed the PANAS again. For most participants (38 of 56 participants), the cognitive and MRI sessions were scheduled on two separate days (median number of days between the two measurement sessions: 1 , interquartile range: 2 , in four occasions the two sessions had to take place in different cycles, keeping cycle phase constant). If two separate measurement sessions were not possible due to a participant's time constraints, the cognitive and MRI sessions were scheduled one after another on the same day, keeping their order constant across participants.

\subsection{Sample description and timing of measurement waves}

For all naturally cycling women (participants in the pill group at T1 as well as participants in the control group at $\mathrm{T} 1$ and $\mathrm{T} 2$ ), measurements were scheduled in the early follicular phase (day 1-10 after begin of menses, mean cycle day: $5.6( \pm 3.3))$. For women in the pill group

Table 1

Sample characteristics.

\begin{tabular}{lll}
\hline Variable & Pill group & Control group \\
\hline Age (in years) & $21.25( \pm 4.02)$ & $21.5( \pm 2.82)$ \\
Years of education & $14.31( \pm 3.2)$ & $14.68( \pm 2.46)$ \\
Participants who ever used & 15 & 20 \\
HC & & \\
Previous HC use (in & $43.79( \pm 55.8$, median $=$ & $43.95( \pm 32.9$, median $=$ \\
$\quad$ months) & $29)$ & $39)$ \\
\hline
\end{tabular}

HC = hormonal contraceptives; "Previous HC use" for women who had ever used hormonal contraceptives. 


\section{Study Design}

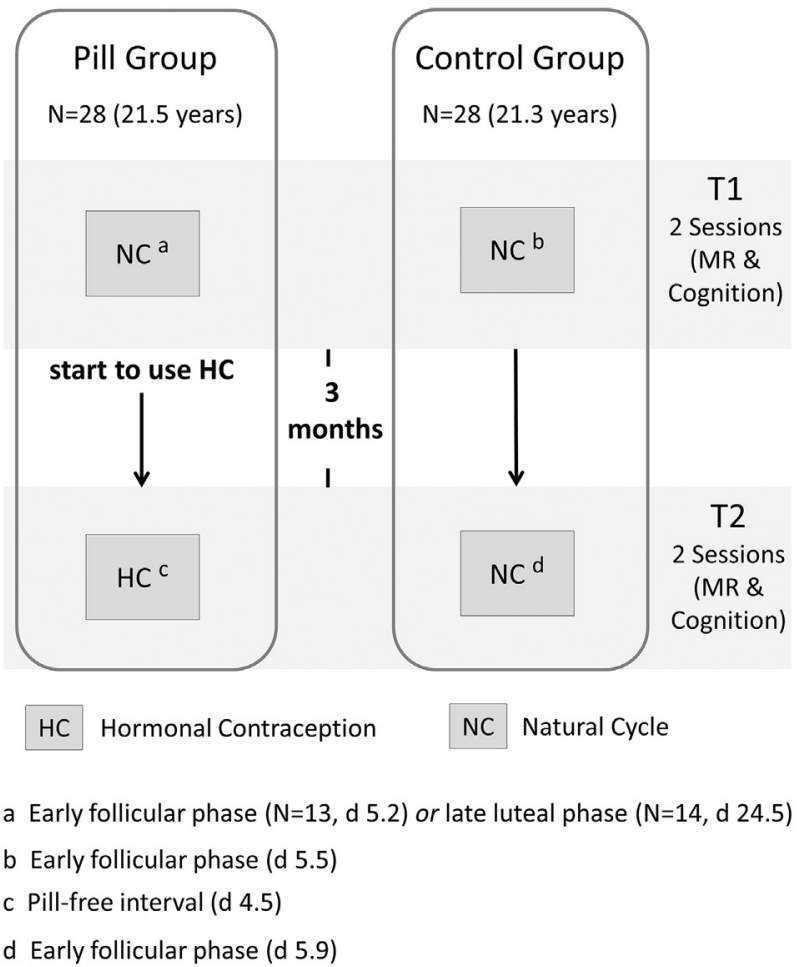

Fig. 1. Overview of study design and procedure. $d=$ mean cycle day (day $1=$ first day of menses or first pill-free day).

who wanted to start using the pill in the following cycle, T1 was scheduled to take place in the late luteal phase (at the end of the cycle). Thirteen women in the pill group were measured in the early follicular phase of their menstrual cycle for at least one T1 measurement session (mean cycle day $5.2( \pm 3.4)$ ). Fourteen women in the pill group were measured in the late luteal phase at T1 (mean cycle day: $24.5( \pm 3.4)$ ). For one participant in the pill group, it was not possible to determine her cycle phase at T1 due to an irregular cycle. Since the late luteal and early follicular cycle phases follow one another and are relatively similar with regard to hormonal levels, they should result in relatively similar states of brain structure with regard to monthly alterations. This assumption was supported by our data: Participants in the pill group who were measured in the follicular or luteal phase at T1 did not differ with regard to our variables of interest, namely, positive and negative affect scores and gray matter volume in the cluster derived from the VBM analysis (two-sample t-tests all $t<1, p>0.1$ ). At T2, women in the pill group were measured in the pill-free interval (mean day: 4.46 ( \pm 2.03 ), with day 1 being defined as the first pillfree day). Women in the control group were measured in the early follicular phase at both time points (mean cycle day T1: $5.5( \pm 3.1)$, mean cycle day T2: $5.9( \pm 3.3))$.

The mean interval between T1 and T2 in the pill group and the control group was about three months (interval in days for pill group: 97.3 ( \pm 14.8$)$, for control group: $97.7( \pm 25.1)$ ). For one woman in the pill group, T2 took place after two rather than three months of regular pill use, and another was tested after four rather than three months. One woman used a continuous pill (that did not contain pill-free intervals) and was therefore tested during pill use at T2. The MR scan at T2 had to be delayed by 3 months for one woman in the control group, leading to a prolonged interval between the first and second MR scan. Excluding these participants from the VBM analysis did not influence the main outcome. The use of parametric tests was justified by tests of normality and variance homogeneity as well as visual inspection of data distribution and standardized residual distribution for the linear models. Where these assumptions were not met, non-parametric tests were used.

All participants in the pill group used combined oral contraceptive pills during the study phase, except for two women using a progesterone-only pill. The distribution of pill types and progestin components was as follows: 14 participants used 2 nd generation pills (Levonorgestrel: $n=12$; Desogestrel: $n=2$ ) and 14 participants used 4th generation pills (Dienogest: $n=10$; Chlormadinonacetat: $n=2$; Drosperinon: $n=2$ ).

\subsection{Hormonal assessment}

Saliva samples were collected using SaliCap collection devices (IBL International, Hamburg, Germany), which are validated for the sampling of steroid hormones. To account for the pulsative secretion of estrogen, we collected three samples spread over the respective session and pooled them afterwards, to minimize the effect of short-term fluctuations of hormone concentrations. Immediately after collection, the saliva samples were frozen and stored at $-25^{\circ} \mathrm{C}$. The estrogen concentrations were determined by a commercial company (IBL International, using a saliva immunoassay $17 ß$-Estradiol ELISA kit). The hormonal data at T1 for one participant in the control group were missing (estrogen and progesterone levels), and estrogen levels at T2 could not be determined for another participant in the pill group. Hormonal levels were log-transformed to correct for non-normal distributions, but due to variance inhomogeneity between the groups at T2 in estrogen levels, estrogen levels were analyzed by means of non-parametric tests. Paired ttests (Wilcoxon signed-rank tests) were used to assess change in estrogen and progesterone across the two measurement time points for each group. Interaction effects were tested by direct comparison of two mixed-linear models (with and without the time-by-group interaction term) in R using the anova function. The influence of cycle phase was assessed by conducting a two-sample t-test (Wilcoxon rank-sum test) comparing hormonal levels of women in the pill group at T1 who were measured during the early phase of their cycle with those assessed in the late phase of their cycle.

\subsection{Affect data}

The PANAS (Watson et al., 1988) includes 20 positive and 20 negative mood adjectives. For each item, participants indicated to what degree they were momentarily feeling that way on an eight point Likertscale (ranging from 1 "does not fit at all" to 8 "fits very well"). The questionnaire was administered at $\mathrm{T} 1$ and $\mathrm{T} 2$ during both the cognitive and the MRI sessions. Positive affect (PA) was determined as the mean value for the positive items and negative affect (NA) for negative items respectively. The scale had very good internal consistency (Cronbachs $\alpha$ $\mathrm{PA}$ at T1 $(\mathrm{T} 2)=0.94(0.95), \mathrm{NA}$ at T1 $(\mathrm{T} 2)=0.94(0.95))$. If possible, mean scores were calculated from the two questionnaires at each time point, thus yielding measures of participants' average (habitual) affect across the respective testing situations. Visual inspection of the distribution of NA values indicated that individuals tended to be clustered at the low end of the scale, resulting in a restriction of range at both time points (skewness at $\mathrm{T} 1=1.59$, at $\mathrm{T} 2=1.75$, median at $\mathrm{T} 1=1.40$, at $\mathrm{T} 2=1.55)$. Therefore, non-parametric tests were used for NA in all analyses. Paired t-tests (Wilcoxon signed-rank tests) were used to assess change in PA and NA across T1 and T2 for each group. Interaction effects were tested by directly comparing two mixed-linear models (with and without the time-by-group interaction term) in R using the anova function. The results of this analysis should be interpreted with caution for NA, because the standardized residuals of the model deviated from normality. The influence of cycle phase was assessed by conducting two-sample t-tests (Wilcoxon rank-sum tests) between T1 scores of women in the pill group measured during early or late cycle phases. 


\subsection{Cognitive tasks}

To assess whether hormonal contraceptive intake is accompanied by cognitive alterations, we included a number of cognitive tasks at both measurement time points. We used three tasks assessing episodic verbal memory: word-list recall (Schmiedek et al., 2010), word-nonword cued recall (Mårtensson and Lövdén, 2011), and face-name cued recall (Mårtensson and Lövdén, 2011); one task assessing working memory: dual-2-back (Jaeggi et al., 2010); and one task measuring object location memory (Schmiedek et al., 2010). In addition, tasks measuring spatial abilities were applied, assessing mental rotation (Vandenberg and Kuse, 1978), navigation (Gramann et al., 2005), spatial orientation (Guilford and Zimmerman, 1948), and perspective taking (Hegarty, 2004). Executive functioning was assessed with a taskswitching paradigm (King et al., 2007). All tasks except for the Guilford-Zimmerman spatial orientation task were computer-based. Details on the tasks can be found in the corresponding reference articles. Group-by-time interactions in task performance were analyzed in a linear mixed-effects model including age as a covariate of no interest. A pvalue of $p<0.005$ was determined by Bonferroni correction to control for multiple testing.

\subsection{MRI data acquisition}

MRIs were acquired using a 3T Magnetom Tim Trio MRI scanner system (Siemens Medical Systems, Erlangen, Germany) using a 12-channel radiofrequency head coil. High-resolution anatomical images were collected using a T1-weighted 3D MPRAGE sequence $(\mathrm{TR}=2500 \mathrm{~ms}$, TE $=$ $4.77 \mathrm{~ms}, \mathrm{TI}=1100 \mathrm{~ms}$, acquisition matrix $=256 \times 256 \times 192$, sagittal FOV $=256 \mathrm{~mm}$, flip angle $=7^{\circ}$, voxel size $=1.0 \times 1.0 \times 1.0 \mathrm{~mm}^{3}$ ). Whole-brain functional images were acquired using a T2*-weighted echo-planar imaging (EPI) sequence sensitive to blood-oxygenationlevel dependent (BOLD) contrast $(\mathrm{TR}=2000 \mathrm{~ms}, \mathrm{TE}=30 \mathrm{~ms}$, image matrix $=72 \times 72, \mathrm{FOV}=216 \mathrm{~mm}$, flip angle $=80^{\circ}$, slice thickness $=$ $2.0 \mathrm{~mm}$, distance factor $=20 \%$, voxel size $3.0 \times 3.0 \times 3.0 \mathrm{~mm}^{3}, 36$ axial slices). 150 image volumes aligned to AC-PC were acquired. Participants were instructed to keep their eyes closed and relax during this resting state measurement.

\subsection{MRI data analysis}

\subsubsection{Analysis of structural data}

Anatomical data were processed by means of the VBM8 toolbox (http://dbm.neuro.uni-jena.de/vbm.html) with default parameters by Gaser and the SPM8 software package (http://www.fil.ion.ucl.ac.uk/ $\mathrm{spm})$. The VBM8 preprocessing involves bias correction, tissue classification, and registration. The "nonlinear only" modulation was applied in order to preserve the volume of a particular tissue within a voxel by multiplying voxel values in the segmented images by the Jacobian determinants derived from the spatial normalization step. In effect, the analysis of modulated data tests for regional differences in the absolute amount (volume) of gray matter. Images were smoothed with a fullwidth half-maximum kernel of $8 \mathrm{~mm}$. Statistical analysis was carried out by means of a whole-brain group-by-time interaction using the flexible factorial model implemented in SPM8. Age, total intracranial volume, estrogen, and progesterone levels (the latter two logtransformed) were entered as covariates of no interest. For two single observations, no estrogen and/or progesterone levels were available. These data points were replaced by the mean of the respective group and time point. The resulting maps were thresholded using $p<0.001$, and a cluster extent threshold was combined with a non-stationary smoothness correction (Hayasaka and Nichols, 2004). The cluster extent threshold was determined by Monte Carlo simulation using the program AlphaSim (Ward, 2000). The cluster size (>198 voxels) was chosen such that the overall probability (combining statistical extent and cluster size threshold) of the type-I error was below 0.01. After computing the whole-brain analysis, the gray matter volumes in the statistically significant regions were extracted by means of rex (https://www.nitrc.org/projects/rex/) for each participant.

\subsubsection{Analysis of functional resting state data}

The first ten EPI volumes were discarded to allow the magnetization to approach a dynamic equilibrium. The data pre-processing, including slice timing, head motion correction (with a least squares approach and a 6-parameter spatial transformation), and spatial normalization to the Montreal Neurological Institute (MNI) template (resampling voxel size of $3 \times 3 \times 3 \mathrm{~mm}^{3}$ ), were conducted using the SPM8 and Data Processing Assistant for Resting-State fMRI (DPARSF; Chao-Gan and Yu-Feng, 2010). A spatial filter of $4 \mathrm{~mm}$ FWHM was used. Participants with head motion above $2 \mathrm{~mm}$ of maximal translation (in any direction of $\mathrm{x}, \mathrm{y}$, or $\mathrm{z}$ ) and $2.0^{\circ}$ of maximal rotation throughout the course of scanning were excluded (one participant). After pre-processing, linear trends were removed. The fMRI data were then temporally bandpass filtered $(0.01-0.08 \mathrm{~Hz})$ to reduce low-frequency drift and highfrequency respiratory and cardiac noise (Biswal et al., 1995). To reduce the effect of physiological artifacts, we removed effects of the following nuisance covariates: global mean signal, the six motion parameters, signal from cerebrospinal fluid and white matter (Fox et al., 2005; Kelly et al., 2008). A whole-brain group-by-time interaction analysis was performed by means of the flexible factorial model implemented in SPM8. Age was entered as a covariate of no interest. The resulting maps were thresholded using $p<0.005$, and a cluster extent threshold was combined with a non-stationary smoothness correction (Hayasaka and Nichols, 2004). The cluster extent threshold was again determined by Monte Carlo simulation using the program AlphaSim (Ward, 2000). The cluster size (>15 voxels) was chosen such that the overall probability (combining statistical extent and cluster size threshold) of the type-I error was below 0.01 .

\section{Results}

\subsection{Hormonal data}

Means and standard deviations for hormonal data of each group and time point are reported in Table 2. Estrogen levels did not differ between the two time points, neither for the pill group nor for the control group, and no interaction between group and time was observed (all $p>0.1$ ). Estrogen levels did not differ between women in the pill group who were measured during the late compared to those measured during the early cycle phase $(p>0.05)$. Progesterone levels in the pill group, but not in the control group, decreased significantly from T1 to T2 (pill group $t(27)=2.41, p=0.02$, control group $p>0.1$; group-bytime interaction $\left.\chi^{2}(1, N=55)=4.58, p=0.032\right)$. Also, progesterone levels in the pill group were significantly higher at T1 than levels in the control group at $\mathrm{T} 1(t(52)=2.23, p=0.03)$. We attribute the raised progesterone levels in the pill group at $\mathrm{T} 1$ to the fact that some women in this group had to be measured at the end of the luteal phase, where progesterone levels are falling but may not yet have reached their lowest levels. Indeed, a two-sample t-test revealed significantly higher

Table 2

Salivary hormonal levels $(\mathrm{pg} / \mathrm{mL})$ for both groups and time points.

\begin{tabular}{lrr}
\hline & Time point 1 & Time point 2 \\
\hline Estrogen & & \\
$\begin{array}{l}\text { Pill group } \\
\text { Control group }\end{array}$ & $15.88( \pm 14.6)$ & $17.48( \pm 15.9)$ \\
Progesterone & $13.03( \pm 14.0)$ & $9.65( \pm 10.9)$ \\
$\begin{array}{l}\text { Pill group } \\
\text { Control group }\end{array}$ & & \\
\hline
\end{tabular}

Values represent means ( \pm standard deviations). 
progesterone levels in the participants in the pill group who were measured during the late luteal compared to those measured in the early follicular cycle phase $(t(26)=2.14, p=0.04)$.

\subsection{Affect data}

PA and NA scores for both groups are reported in Table 3. The PA and NA scores of participants in the pill group who were measured in the follicular phase at T1 did not differ from those of participants measured in the luteal phase (both $p>0.1$ ). PA significantly decreased from T1 to T2 in the pill group $(t(27)=2.38, p=0.025)$, but not in the control group $\left(t(27)<1 ; p>0.1\right.$; group-by-time interaction $\chi^{2}(1, N=56)=$ $3.02, p=0.082)$. The individual changes in PA scores are shown in Fig. 2. The plot highlights that not all women in the pill group experienced a decrease in PA (some also showed an increase) and that the extent of PA decrease is quite variable across individuals in the pill group. To examine possible factors explaining these differences within the pill group, we correlated the change scores with age and pill progestin concentration (in mg) using the Pearson product-moment correlation and compared change with regard to previous pill use (yes/no) and generation of progestin pill ( $2 \mathrm{nd} / 4$ th $)$ by means of two-sample t-tests. A significant correlation between change in PA and age was observed $(r(26)=$ $0.42, p=0.028$, Fig. 3 ; see Supplementary material for results of a multiple regression analysis including all variables). The younger the women in the pill group were, the stronger their PA decreased from before to after onset of hormonal contraception. Previous pill use, generation of progestin pill, and progestin concentration were not associated with change in PA.

With regard to NA, both groups did not change significantly from T1 to T2 (all $p>0.1$ ). Change in NA in the pill group correlated with age (Spearman's rho(26) $=-0.43 p=0.023$ ). That is, younger women in the pill group showed greater reductions in PA and increases in NA from $\mathrm{T} 1$ to $\mathrm{T} 2$.

\subsection{Cognitive data}

No significant main effect of group or group-by-time interaction was observed in performance of any of the cognitive tasks (all $p>0.005$ ).

\subsection{MR data}

\subsubsection{Structural MR data}

The whole-brain group-by-time interaction analysis in VBM revealed a single cluster in the left amygdala/anterior parahippocampal gyrus (PHG) $(-12,-6,-26$, size: 539 voxels, $p<0.001, \mathrm{k}>189$ voxels, mean $\mathrm{t}$-value for voxels in the significant cluster $=3.50$; see Fig. 4 ). The interaction was driven by a volume decrease in the pill group whereas the mean level of the control group did not change. This is shown in Fig. 5 where the individual gray matter volume change scores of that region are plotted. Participants in the pill group who were measured in the follicular cycle phase at T1 did not differ from participants measured in the luteal phase with regard to extracted gray matter volumes $(t<1, p>0.1)$. Change in extracted gray matter in the pill group

Table 3

Positive and negative affect scores for both groups and time points.

\begin{tabular}{llc}
\hline & Time point 1 & Time point 2 \\
\hline $\begin{array}{l}\text { Positive affect } \\
\text { Pill group }\end{array}$ & $4.91(1.0)$ & $4.39(1.2)$ \\
Control group & $5.07(0.9)$ & $5.00(0.8)$ \\
Negative affect & & \\
Pill group & $1.81(0.8)$ & $1.98(0.9)$ \\
Control group & $1.63(0.8)$ & $1.61(0.7)$ \\
\hline
\end{tabular}

Means (standard deviations) for positive and negative scores (range 1-8).

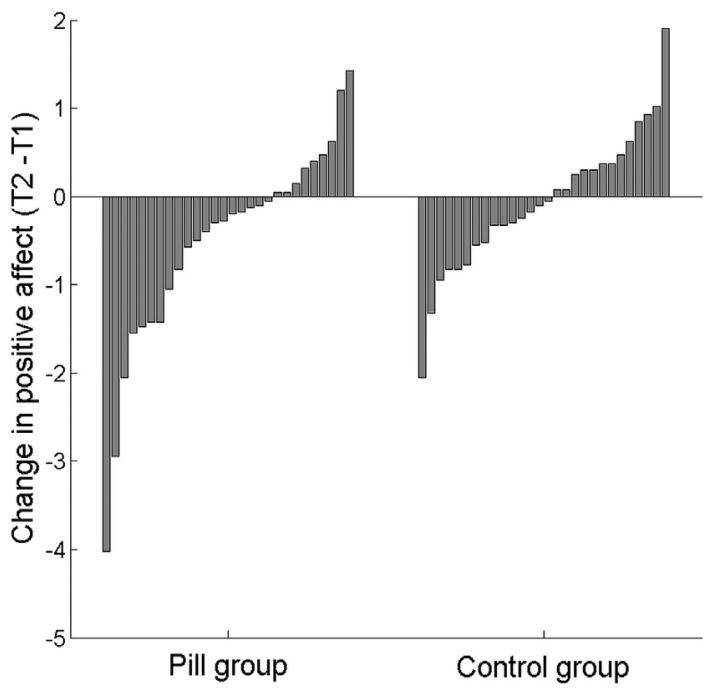

Fig. 2. Individual change scores for positive affect. Negative values represent a decrease and positive values an increase in positive affect.

was not associated with previous pill use, generation of progestin, or progestin concentration of the pill used (Pearson product-moment correlations and two sample t-tests, all $p>0.1$; see Supplementary material for results of a multiple regression analysis including all variables). The cluster was still significant when the analysis was repeated without the two women taking progestin-only pills.

\subsubsection{Resting state functional connectivity data}

Functional connectivity maps with a seed region (mean BOLD in seed) in the left amygdala/anterior PHG cluster resulting from the VBM interaction analysis were used to conduct an interaction analysis testing for a group-by-time interaction in functional connectivity of the structural region of interest ( $p<0.005, \mathrm{k}>15$ voxels). Due to the relatively liberal $p$-threshold, the result should be interpreted with caution. A significant interaction was observed between the left amygdala/anterior PHG cluster and a cluster in the right dorsolateral prefrontal cortex (DLPFC, MNI coordinates 48,21,21; see Fig. 6). The cluster was still significant when estrogen and progesterone levels were included in the analysis as covariates of no interest. Mean functional connectivity $(\mathrm{FC}$ ) between the two regions changed from positive

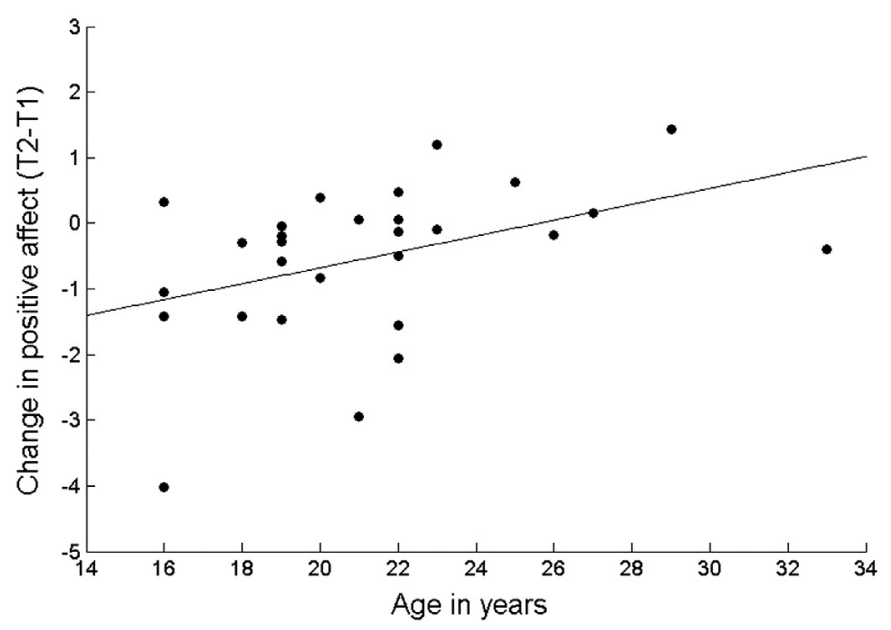

Fig. 3. Age correlates positively with change in positive affect in women in the pill group $(r(26)=0.42, p=0.028)$ 


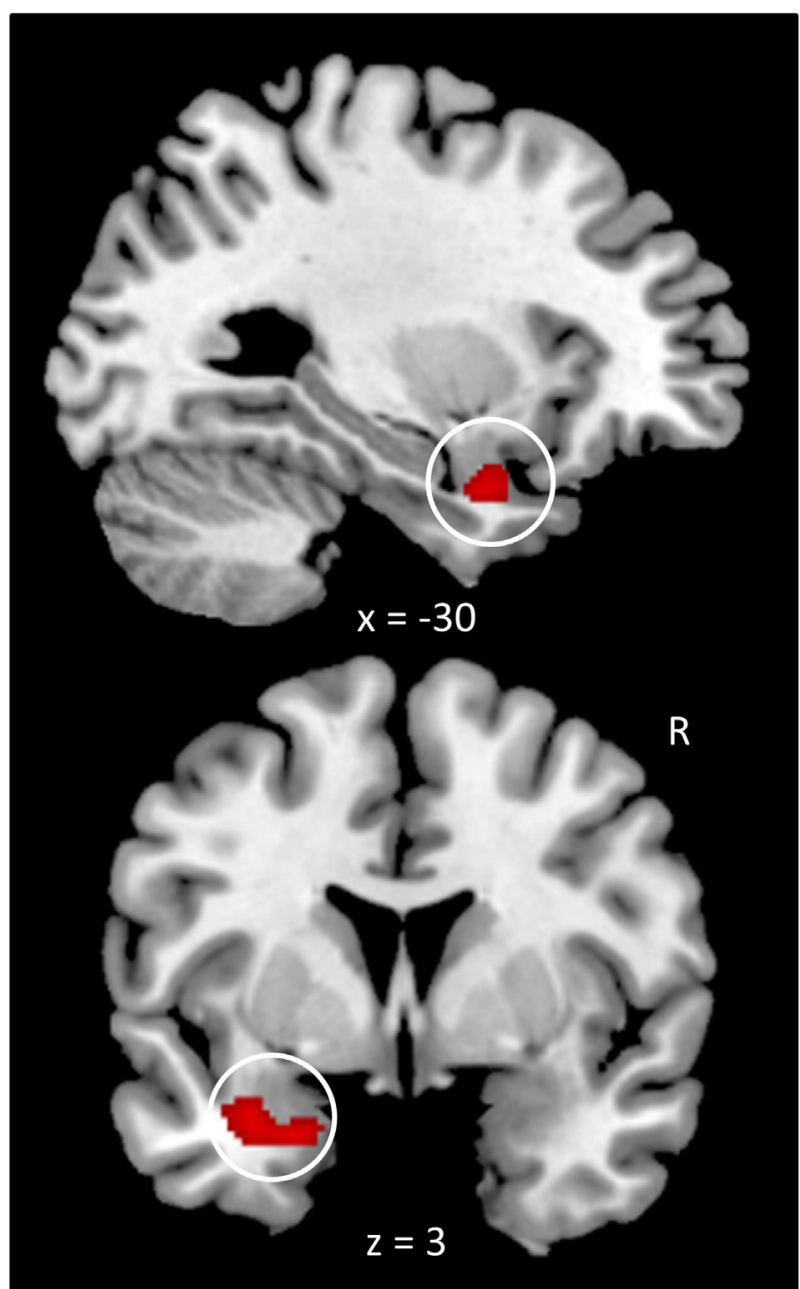

Fig. 4. The left amygdala/anterior parahippocampal gyrus emerged as the only significant cluster in a whole-brain group-by-time interaction analysis $(p<0.001, \mathrm{k}>200$ voxels, corrected for non-stationary smoothness, significant cluster is surrounded by white circle). Within this region, gray matter volume decreased in the pill group and did not show mean level change in the control group. Coordinates refer to MNI space.

to negative connectivity in the pill group (mean (standard deviation) FC at T1 $=0.003(0.15)$, at T2 $=-0.089(0.15)$, whereas the opposite held for the control group ( $\mathrm{FC}$ at T1 $=-0.076(0.13)$, at T2 $=0.043(0.13)$.

\subsection{Brain-affect associations}

We further inspected potential associations of PA (decreasing from $\mathrm{T} 1$ to $\mathrm{T} 2$ in the pill group) and gray matter volume in the left amygdala/anterior PHG (decreasing in parallel in the pill group). We correlated the PA change scores with gray matter volume change scores within participants in the pill group and performed a multiple regression to predict PA by gray matter volume in the entire sample at T2, while controlling for the group differences. Change in PA and gray matter volume was not significantly correlated in women in the pill group $(p>.05)$. A linear multiple regression was used to investigate whether gray matter volumes predicted PA at T2 beyond the effect of group membership. Age and group were included as covariates and the model with and without gray matter volume as a predictor were compared using the anova function in R. Gray matter volume in the amygdala/anterior PHG significantly increased the predictive value of the model $(F(1,52)=5.71, p=0.021$, estimate: 7.05$)$. Greater gray matter volume was associated with more PA at T2 in the entire sample.

\section{Discussion}

The present longitudinal study investigates brain structural alterations after three months of oral contraceptive intake. We found that the left amygdala/anterior PHG volume decreased in women using hormonal contraceptives as compared to a control group of women who did not take contraceptives. We also observed a significant group-bytime interaction in functional connectivity of this region with the right DLPFC. This is the first indication that hormonal contraceptive use is accompanied by decreases in gray matter volume in a group of healthy young women within a time span of only a few months. The localization of the gray matter reductions in the amygdala matches previous literature on hormone-related brain plasticity and suggests links to mood alterations in women using hormonal contraceptives. Indeed, PA decreased in the pill group on average, and PA was positively related to gray matter volume in the amygdala/anterior PHG at T2 across both groups.

The groups did not differ systematically in cognitive performance changes. This observation adds to the research field studying female cognitive performance variations in relation to hormonal status. Two recent reviews emphasize the inconsistency of findings on the effects of hormonal contraception on cognition (Gogos et al., 2014; Warren et al., 2014). Together with our findings, this suggests that any potential effects are weak, restricted to very specific cognitive processes, or are highly variable between individuals. However, our study design was not able to detect effects of long-term hormonal contraception on cognitive performance. Hence, effects of prolonged endogenous hormone exposure on cognitive performance cannot be excluded.

None of the previous cross-sectional MR studies observed differences in amygdala/anterior PHG volume between hormonal contraceptive users and non-users (De Bondt et al., 2013; Petersen et al., 2015; Pletzer et al., 2010; Pletzer and Kerschbaum, 2014). A comparison of the present findings with the previous literature on gray matter variation is difficult because we took a longitudinal approach rather than applying the previously used cross-sectional study design. The differences in design also led to variations in durations of contraceptive use: longterm users taking contraceptives for several years were usually included in cross-sectional analyses, whereas we focused on effects of contraceptives over the course of a few months. The inconsistent results could indicate several distinct neural change processes that differ depending on the duration of contraception intake. The amygdala might be very sensitive to the hormonal change processes occurring soon after the onset of hormonal contraception. A potential renormalization of the structural changes under continuous hormonal contraceptive use could explain why cross-sectional studies have not observed differences between (long-term) contraceptive users and non-users. Our experimental

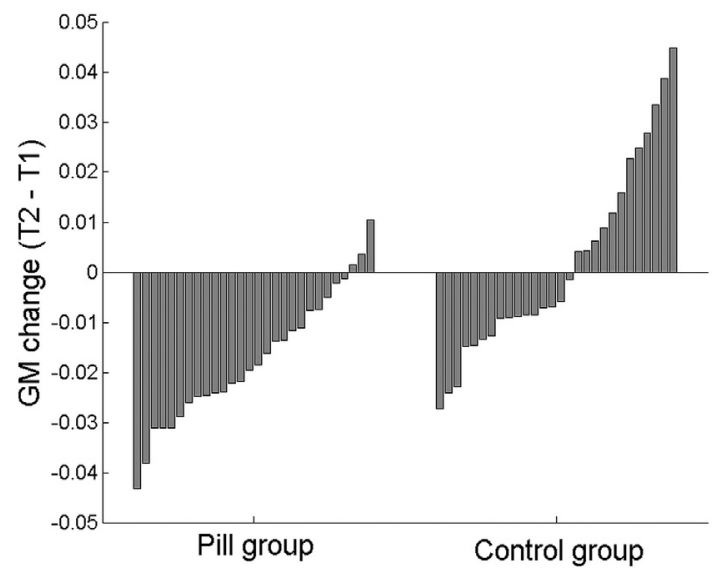

Fig. 5. Individual change scores for gray matter (GM) volume in the amygdala/anterior parahippocampal gyrus. 


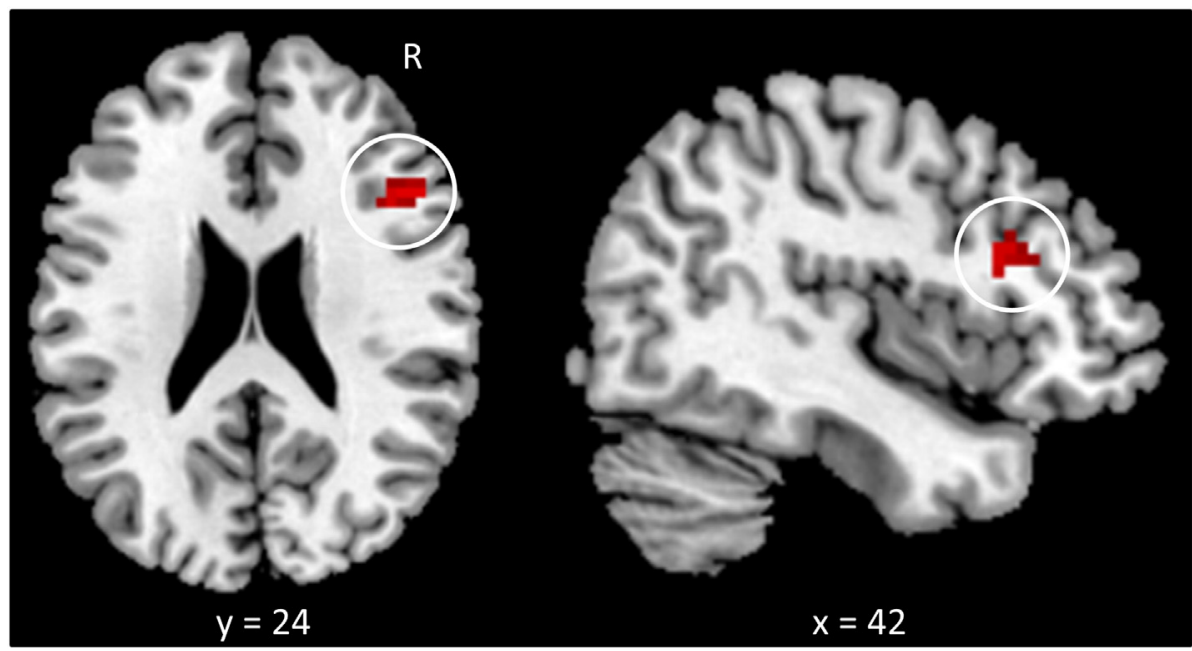

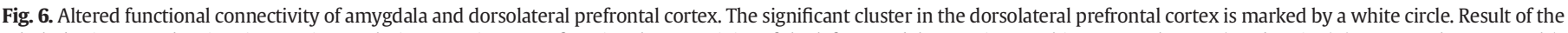

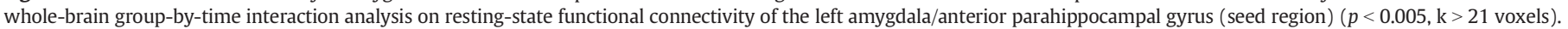
Coordinates refer to MNI space.

design did not allow extrapolation of the functional form of the observed brain structure changes, such as long-term mean trends, reversals, or oscillations. Other study characteristics (e.g., region-of-interest vs. whole-brain analyses) and especially characteristics of the samples (e.g., age, cultural background, pill types used) could contribute to the inconsistency of findings as well. This emphasizes the need for future, more fine-grained investigation of gray matter changes under hormonal contraception.

Our findings are in line with studies showing a special sensitivity of the amygdala to female gonadal hormones (Van Wingen et al., 2011; for an overview on functional studies, see Lisofsky et al., 2015b). Previous cross-sectional and longitudinal studies observed altered amygdala reactivity in hormonal contraceptive users as compared to naturally cycling women (Gingnell et al., 2013; Petersen and Cahill, 2015). Altered amygdala reactivity and functional connectivity with the medial prefrontal cortex (PFC) was also observed in naturally cycling women after a single dose of progesterone (Van Wingen et al., 2007, 2008). To date, longitudinal evidence on structural changes following contraceptive intake was lacking. Our results are based on a hypothesis-free whole-brain analysis and strongly suggest that hormonal contraceptives affect not only amygdala function, but also amygdala morphology. The observed gray matter volume decreases were consistent across individuals and their magnitude was not associated with age, previous hormonal contraceptive use, progestin concentration, or type of progestin component in the pill.

The whole-brain gray-matter analysis revealed a lateralized finding relating to amygdala/anterior PHG volume change. The left amygdala/ anterior PHG decreased within the first months of pill use, whereas there was no interaction in right amygdala volume at the same statistical threshold (a bilateral effect only appeared if the threshold was lowered to $p<0.005, \mathrm{k}>50$ ). This could indicate a lateralization difference in the effect of hormonal contraceptives on brain structure. With regard to brain function, lateralization of the amygdala/hippocampal complex has been observed in relation to the natural fluctuation of female gonadal hormones (Lisofsky et al., 2015b). In the concordance analysis of previous task-based fMRI studies, stronger left-lateralized activity was observed during the luteal phase (high estrogen and progesterone) compared to the follicular phase (low estrogen and progesterone) of the natural menstrual cycle. It is difficult to relate these two findings, because the former is based on brain function in naturally cycling women, whereas the present finding relates to the effect of hormonal contraceptives on brain structure. Nonetheless, both findings indicate some lateralized neural effects of estrogen and progesterone, requiring further investigation.

Following up on the structural results, we investigated whether we would also see indications of plasticity at the functional level. For this purpose we focused on the functional connectivity of the amygdala/anterior PHG. We observed a significant group-by-time interaction in functional connectivity between the seed region and the right DLPFC. Note, however, that this should be interpreted with caution because the cluster in the DLPFC was comparatively small. Nevertheless, the observation is in line with the hypothesis of hormone-related changes in the brain's emotion regulation system during contraceptive use. In conjunction with the amygdala, the DLPFC facilitates emotion regulation (Ochsner et al., 2012). At the same time DLPFC activity has also been shown to be hormone-sensitive. Amin et al. (2006) reported that DLPFC activity in response to emotional stimuli correlated with estrogen levels in naturally cycling women. The observed differences in functional connectivity between amygdala and DLPFC could therefore also be due to more subtle, short-term differences in hormonal states between the measurement phases. The direction of the interaction effect indicates a switch from positive to negative connectivity in women who started using hormonal contraceptives whereas the opposite held for women in the control group. Both positive and negative connectivity have been described between amygdala and frontal cortices (Gee et al., 2013; Hariri et al., 2003; Kim et al., 2011b; Kim et al., 2011a), but the neurobiological basis for positive in contrast to negative connectivity (e.g., whether one indicates inhibitory processes) remains unclear. In a previous study by Kim et al. (2011b), negative resting-state functional connectivity between the amygdala and the medial PFC was related to high self-reported anxiety, whereas participants with low anxiety showed positive functional connectivity between these regions (Kim et al., 2011b). The potential link between amygdala connectivity induced by hormonal contraception and behavioral outcomes, such as anxiety, should be investigated in future studies.

Besides the neuroimaging findings, we also observed change in current mood within the pill group. On average, PA decreased, with a large range of variability at the individual level. However, the potential causes of this mood perturbation remain ambiguous: They could be viewed as indicative of actual mood changes induced by hormonal contraceptives, but placebo effects and expectations about side-effects could also play a role. Why mean changes in mood were restricted to PA is an open question. The restriction of range in NA might have hindered the detection of changes across time. Given this limitation, we cannot determine 
whether mood changes following hormonal contraceptive intake are restricted to PA, as suggested by previous experimental work (Jarva and Oinonen, 2007). In line with previous results, changes in PA and NA in the pill group correlated negatively with age. Women below the age of 20 might be especially susceptible to negative mood effects following hormonal contraceptive intake, while women above the age of 30 may rather experience positive mood effects (Oinonen and Mazmanian, 2002). The underlying factors causing this age difference have not been investigated. Possible explanations could be age differences in the expectations regarding side-effects of the pill, age differences in coping with mood swings, age differences in overall affect variability, or a selection effect in the sense that women who experience negative side-effects of pill use, including decreases in PA, are more likely to switch to other contraceptives than women who do not experience such side-effects. These potential explanations could be a fruitful topic for future studies.

The variability in affective change is very likely not only attributable to age differences or effects mediated by age. We can only speculate about other factors causing the differences in affective change: Following up on the findings reported by Gingnell et al. (2013), previous (negative) experience with mood changes during hormonal contraception might be related to mood swings in later phases of contraception. Starting to use hormonal contraception might also accompany other change processes (e.g., physiological changes such as weight gain, or social changes such as a new partnership), which can in turn impact individual affect. Short-term affective fluctuations and diverse re-test effects may also need to be considered in this context (and would apply to change in affect in the control group as well). The present study shows that even in the presence of similar neural alterations (i.e., decreases in gray matter volume), psychological outcomes (i.e., mood) do not change equally across individuals.

Beyond parallel changes in brain structure and mood, we found evidence that affect correlated with the brain region in which contraception-induced morphological changes were observed. Greater gray matter volume in the amygdala/anterior PHG was associated with more PA in the entire sample after the intervention. At T2, the groups differed significantly in PA (but not in NA). Gray matter volume significantly enhanced the predictive power of the model beyond this effect of group. Thus, some variance of the inter-individual differences in PA was explained by inter-individual differences in gray matter volume in the region showing decreases in the pill group. Greater amygdala volume was associated with more PA in the entire sample. These exploratory findings need to be replicated and should be interpreted with caution until then. The finding indicates an association between amygdala structure and affect, in line with previously reported associations between smaller amygdala volumes and mood disorders (Hamilton et al., 2008). The amygdala is involved in up- and downregulation of positive as well as negative emotions (e.g., Kim and Hamann, 2007; Ochsner et al., 2004), and some investigations have revealed stronger amygdala reactivity for positive compared to negative affect regulation (Kim and Hamann, 2007). We did not observe a significant correlation between the magnitude of gray matter volume changes and affective change in the pill group. While the gray matter volume decreases were very consistent across participants in the pill group, affective change varied substantially between participants. Thus, further factors that moderate and mediate the effects of structural amygdala alterations on affect need to be considered.

We can only speculate about the neurobiological mechanisms that may have caused the observed amygdala volume reductions. The whole-brain interaction analysis was corrected for saliva estrogen and progesterone levels at the measurement phase. Therefore, the effects are unlikely to reflect the momentary hormonal environment, but rather represent the influence of chronically altered hormone levels in the pill group. The amygdala is characterized by a high density of estrogen receptors (Österlund and Hurd, 2001; Österlund et al., 2000). Progesterone metabolites can also modulate $\Upsilon$-aminobutyric acid (GABA) receptors and influence the amygdala via this route (Akwa et al., 1999; Majewska et al., 1986; Van Wingen et al., 2008). Thus, external estrogen and progesterone administered via contraceptive pills may act on the steroid-sensitive receptors in the amygdala and change cell morphology and function. At the same time, endogenous estrogen, progesterone, and testosterone levels are lowered during use of hormonal contraceptives (Hietala et al., 2007; Sahlberg et al., 1987; Wiegratz et al., 2003). Animal studies have shown that estrogen, progesterone, and combined estrogen and progesterone treatment all lead to increases in neuronal and somatic volume in the posterodorsal medial amygdala in ovariectomized female rats (De Castilhos et al., 2010). Hormone-induced changes in cell survival and proliferation, increases in dendritic spine density, dendrite length, and dendritic branching have been proposed as neurobiological causes for these volume changes (Cooke and Woolley, 2005). Whether morphological changes are also mediated by functional changes that have been caused by hormonal alterations (e.g., hyperreactivity) remains to be investigated. For instance, functional neuroimaging studies in humans have documented that progesterone administration (without additional estrogen) induces increased amygdala reactivity (Van Wingen et al., 2008). Together with data from animal studies, this suggests that the effect of progesterone alone would suffice to initiate functional changes, potentially followed by structural changes in the amygdala. In line with this notion, the two women using progestin-only pills in our sample showed similar gray matter volume decreases as those women using combined contraceptive pills (albeit only single cases).

Morphological changes in the amygdala following contraceptive use could also be related to altered fear extinction learning in relation to gonadal hormones: Fear extinction learning seems to be modified in women using hormonal contraceptives as well as estrogen-treated rats (Graham and Milad, 2013; Merz et al., 2012b; Zsido, 2014). Hormonal effects on amygdala functioning have been proposed as the neural underpinning for these observations, since fear extinction learning relates to amygdala function (Merz et al., 2012a; Zsido, 2014). However, hormone-induced structural changes in the amygdala have not been connected to fear extinction learning and the association of structural and functional amygdala change via hormonal contraception has not been investigated in that context. A better understanding of hormoneinduced structural brain changes and their potential impact on fear extinction learning would be clinically relevant, because impaired fear extinction learning has been associated with the development of anxiety disorders such as post-traumatic stress disorder (PTSD, Milad et al., 2008; Milad et al., 2006). Thus, hormonal contraceptive use might be a risk factor for PTSD and could influence the efficacy of PTSD therapies, as has been suggested previously (Lebron-Milad et al., 2012).

The present study has some limitations that should be overcome in future studies. First, we did not randomly assign participants to the two groups; therefore, pre-existing differences between groups cannot be ruled out as potential reasons for our observations. However, to the extent that our results are based on within-person changes, this limitation primarily affects generalizability, rather than validity. Second, the study did not use a blinded, placebo-controlled design, which may have biased the affective outcome variables. Third, the findings regarding mood changes are based on a questionnaire assessing negative and positive affect in general, rather than focusing on specific facets of affective experience. Future studies should include additional mood-related variables as well as more experience samples for each mood indicator to increase reliability. Functional imaging paradigms (e.g., emotion regulation) should be applied to test associations between structural and functional task-based alterations in the amygdala following hormonal contraception. Fourth, a number of confounding variables and specific aspects of the study design complicated data analysis (e.g., variables that only applied to one time point or one group, such as cycle phase at T1 or pill type). We tried to control for, or at least describe, the effects of these potential confounding variables on our results by applying different analysis approaches such as multivariate analyses including 
covariates of no interest or comparisons of subgroups. Future studies should try to test effects of hormonal contraceptives in more homogenous participant groups (especially with regard to cycle phase at the first measurement time point), which would make post-hoc analyses of confounding factors unnecessary. Relatedly, larger sample sizes with sufficient power to detect differences between sub-groups (such as different pill types or previous hormonal contraceptive use) should be enrolled. Finally, testing women at several intervals during hormonal contraceptive intake would help to investigate the time course of affective and neural changes following hormonal shifts.

\section{Conclusion}

Taken together, this study demonstrates that the amygdala is altered structurally and functionally in young adult women within the first months of hormonal contraceptive use. The present findings are of general importance for imaging research on women. We also report preliminary evidence suggesting that gray matter in the region showing structural alterations is associated with positive affect across individuals and may therefore be relevant for the psychological wellbeing of hormonal contraceptive users. Further investigations need to clarify the association between neural changes and mood changes in the context of hormonal contraception.

\section{Conflict of interest}

The authors declare no conflict of interest.

\section{Acknowledgments}

We are grateful for the assistance of the MRI team at the Max Planck Institute for Human Development in Berlin, consisting of Sonali Beckmann, Nils Bodammer, Thomas Feg, Sebastian Schröder, and Nadine Taube. We also thank our student assistants, especially Philine Dolz, Moritz Heimbach, and Anne Wilkening, for their help with data collection and preparation. Thanks are also due to Julia Delius for editorial assistance.

\section{Appendix A. Supplementary data}

Supplementary data to this article can be found online at http://dx. doi.org/10.1016/j.neuroimage.2016.04.042.

\section{References}

Akwa, Y., Purdy, R.H., Koob, G.F., Britton, K.T., 1999. The amygdala mediates the anxiolytic-like effect of the neurosteroid allopregnanolone in rat. Behav. Brain Res. 106 (1-2), 119-125. http://dx.doi.org/10.1016/S0166-4328(99)00101-1.

Amin, Z., Epperson, C.N., Constable, R.T., Canli, T., 2006. Effects of estrogen variation on neural correlates of emotional response inhibition. Neurolmage 32 (1), 457-464 http://dx.doi.org/10.1016/j.neuroimage.2006.03.013.

Biswal, B., Yetkin, F.Z., Haughton, V.M., Hyde, J.S., 1995. Functional connectivity in the motor cortex of resting. Magn. Reson. Med. 34, 537-541. http://dx.doi.org/10.1002/ mrm.1910340409.

Chao-Gan, Y., Yu-Feng, Z., 2010. DPARSF: a MATLAB toolbox for "pipeline" data analysis of resting-state fMRI. Front. Syst. Neurosci. 4, 13. http://dx.doi.org/10.3389/fnsys.2010. 00013.

Comasco, E., Frokjaer, V.G., Sundström-Poromaa, I., 2014. Functional and molecular neuroimaging of menopause and hormone replacement therapy. Front. Neurosci. 8 388. http://dx.doi.org/10.3389/fnins.2014.00388.

Cooke, B.M., Woolley, C.S., 2005. Gonadal hormone modulation of dendrites in the mammalian CNS. J. Neurobiol. 64 (1), 34-46. http://dx.doi.org/10.1002/neu.20143.

De Bondt, T., Jacquemyn, Y., Van Hecke, W., Sijbers, J., Sunaert, S., Parizel, P.M., 2013. Regional gray matter volume differences and sex-hormone correlations as a function of menstrual cycle phase and hormonal contraceptives use. Brain Res. 1530, 22-31. http://dx.doi.org/10.1016/j.brainres.2013.07.034

De Castilhos, J., Hermel, E.E.S., Rasia-Filho, A.A., Achaval, M., 2010. Influence of substitutive ovarian steroids in the nuclear and cell body volumes of neurons in the posterodorsa medial amygdala of adult ovariectomized female rats. Neurosci. Lett. 469 (1), 19-23. http://dx.doi.org/10.1016/j.neulet.2009.11.036.
Federal Centre for Health Education (BZgA), 2011. Contraceptive Behaviour of Adults 2011: Results of a Representative Survey. BZgA, Cologne Retrieved from http:// www.bzga.de/pdf.php?id=501 fa1524a1049ef572e0964e085e29b.

Fox, M.D., Snyder, A.Z., Vincent, J.L., Corbetta, M., Van Essen, D.C., Raichle, M.E., 2005. The human brain is intrinsically organized into dynamic, anticorrelated functional networks. Proc. Natl. Acad. Sci. U. S. A. 102 (27), 9673-9678. http://dx.doi.org/10.1073/ pnas.0504136102.

Gee, D.G., Humphreys, K.L., Flannery, J., Goff, B, Telzer, E.H., Shapiro, M. Tottenham, N., 2013. A developmental shift from positive to negative connectivity in human amygdala-prefrontal circuitry. J. Neurosci. 33 (10), 4584-4593. http://dx.doi.org/10. 1523/JNEUROSCI.3446-12.2013.

Gingnell, M., Engman, J., Frick, A., Moby, L., Wikström, J., Fredrikson, M., SundströmPoromaa, I., 2013. Oral contraceptive use changes brain activity and mood in women with previous negative affect on the pill: a double-blinded, placebocontrolled randomized trial of a levonorgestrel-containing combined oral contraceptive. Psychoneuroendocrinology 38 (7), 1133-1144. http://dx.doi.org/10.1016/j. psyneuen.2012.11.006.

Gingnell, M., Bannbers, E., Engman, J., Frick, A., Moby, L., Wikström, J., SundströmPoromaa, I., 2015. The effect of combined hormonal contraceptives use on brain reactivity during response inhibition. Eur. J. Contracep. Reprod. Health Care http://dx.doi. org/10.3109/13625187.2015.1077381 Advance online publication.

Gogos, A., Wu, Y.C., Williams, A.S., Byrne, L.K., 2014. The effects of ethinylestradiol and progestins ("the pill") on cognitive function in pre-menopausal women. Neurochem. Res. 39 (12), 2288-2300. http://dx.doi.org/10.1007/s11064-014-1444-6.

Graham, B.M., Milad, M.R., 2013. Blockade of estrogen by hormonal contraceptives impairs fear extinction in female rats and women. Biol. Psychiatry 73 (4), 371-378. http://dx.doi.org/10.1016/j.biopsych.2012.09.018.

Gramann, K., Müller, H.J., Eick, E.-M., Schönebeck, B., 2005. Evidence of separable spatial representations in a virtual navigation task. J. Exp. Psychol. Hum. Percept. Perform. 31 (6), 1199-1223. http://dx.doi.org/10.1037/0096-1523.31.6.1199.

Guilford, J.P., Zimmerman, W.S., 1948. The Guilford-Zimmerman aptitude survey. J. Appl. Psychol. 32 (1), 24-34. http://dx.doi.org/10.1037/h0063610.

Hamilton, J.P., Siemer, M., Gotlib, I.H., 2008. Amygdala volume in major depressive disorder: a meta-analysis of magnetic resonance imaging studies. Mol. Psychiatry 13 (11), 993-1000. http://dx.doi.org/10.1038/mp.2008.57.

Hariri, A.R., Mattay, V.S., Tessitore, A., Fera, F., Weinberger, D.R., 2003. Neocortical modulation of the amygdala response to fearful stimuli. Biol. Psychiatry 53 (6), 494-501. http://dx.doi.org/10.1016/S0006-3223(02)01786-9.

Hayasaka, S., Nichols, T.E., 2004. Combining voxel intensity and cluster extent with permutation test framework. Neurolmage 23 (1), 54-63. http://dx.doi.org/10.1016/j. neuroimage.2004.04.035.

Hegarty, M., 2004. A dissociation between mental rotation and perspective-taking spatial abilities. Intelligence 32 (2), 175-191, http://dx doi.org/10.1016/jintell.2003.12.001.

Hietala, M., Sandberg, T., Borg, A., Olsson, H., Jernström, H., 2007. Testosterone levels in relation to oral contraceptive use and the androgen receptor CAG and GGC length polymorphisms in healthy young women. Hum. Reprod. 22 (1), 83-91. http://dx.doi.org/ 10.1093/humrep/del318.

Jaeggi, S.M., Studer-Luethi, B., Buschkuehl, M., Su, Y.F., Jonides, J., Perrig, W.J., 2010. The relationship between n-back performance and matrix reasoning: implications for training and transfer. Intelligence 38 (6), 625-635. http://dx.doi.org/10.1016/j.intell.2010.09.001.

Jarva, J.A., Oinonen, K.A., 2007. Do oral contraceptives act as mood stabilizers? Evidence of positive affect stabilization. Arch. Womens Ment. Health 10 (5), 225-234. http://dx. doi.org/10.1007/s00737-007-0197-5.

Johnson, S., Pion, C., Jennings, V., 2013. Current methods and attitudes of women towards contraception in Europe and America. Reprod. Health 10, 7. http://dx.doi.org/10. 1186/1742-4755-10-7.

Jones, J., Mosher, W., Daniels, K., 2012. Current Contraceptive use in the United States, 2006-2010, and Changes in Patterns of Use Since 1995 (National Health Statistics Reports, No. 60). National Center for Health Statistics, Hyattsville, MD Retrieved from http://www.cdc.gov/nchs/data/nhsr/nhsr060.pdf.

Kalmar, J.H., Wang, F., Chepenik, L.G., Womer, Y., Jones, M.M., ... Blumberg, H.P., 2009. Relation between amygdala structure and function in adolescents with bipolar disorder. J. Am. Acad. Child Adolesc. Psychiatry 48 (6), 636-642. http://dx.doi.org/10.1097/CHI. Ob013e31819f6fbc.

Kelly, A.M.C., Uddin, L.Q., Biswal, B.B., Castellanos, F.X., Milham, M.P., 2008. Competition between functional brain networks mediates behavioral variability. Neurolmage 39 (1), 527-537. http://dx.doi.org/10.1016/j.neuroimage.2007.08.008.

Kim, S.H., Hamann, S., 2007. Neural correlates of positive and negative emotion regulation. J. Cogn. Neurosci. 19 (5), 776-798. http://dx.doi.org/10.1162/jocn.2007.19.5.776.

Kim, M.J., Loucks, R.A., Palmer, A.L., Brown, A.C., Kimberly, M., Marchante, A.N., Whalen, P.J., 2011a. From normal emotion to pathological anxiety. Behav. Brain Res. 223 (2), 403-410. http://dx.doi.org/10.1016/j.bbr.2011.04.025.

Kim, M.J., Gee, D.G., Loucks, R.A., Davis, F.C., Whalen, P.J., 2011b. Anxiety dissociates dorsal and ventral medial prefrontal cortex functional connectivity with the amygdala at rest. Cereb. Cortex 21 (7), 1667-1673. http://dx.doi.org/10.1093/cercor/bhq237.

King, J.A., Colla, M., Brass, M., Heuser, I., von Cramon, D., 2007. Inefficient cognitive control in adult ADHD: evidence from trial-by-trial Stroop test and cued task switching performance. Behav. Brain Funct. 3, 42. http://dx.doi.org/10.1186/1744-9081-3-42.

Lebron-Milad, K., Graham, B.M., Milad, M.R., 2012. Low estradiol levels: a vulnerability factor for the development of posttraumatic stress disorder. Biol. Psychiatry 72 (1), 6-7. http://dx.doi.org/10.1016/j.biopsych.2012.04.029.

Lisofsky, N., Mårtensson, J., Eckert, A., Lindenberger, U., Gallinat, J., Kühn, S., 2015a. Hippocampal volume and functional connectivity changes during the female menstrual cycle. Neurolmage 118, 154-162. http://dx.doi.org/10.1016/j.neuroimage.2015.06.012.

Lisofsky, N., Lindenberger, U., Kühn, S., 2015b. Amygdala/hippocampal activation during the menstrual cycle: evidence for lateralization of effects across different tasks. 
Neuropsychologia 67, 55-62. http://dx.doi.org/10.1016/j.neuropsychologia.2014.12. 005.

Majewska, M.D., Harrison, N.L., Schwartz, R.D., Barker, J.L., Paul, S.M., 1986. Steroid hormone metabolites are barbiturate-like modulators of the GABA receptor. Science 232 (4753), 1004-1007. http://dx.doi.org/10.1126/science.2422758.

Mårtensson, J., Lövdén, M., 2011. Do intensive studies of a foreign language improve associative memory performance? Front. Psychol. 2, 12. http://dx.doi.org/10.3389/fpsyg. 2011.00012.

Merz, C.J., Tabbert, K., Schweckendiek, J., Klucken, T., Vaitl, D., Stark, R., Wolf, O.T., 2012a. Neuronal correlates of extinction learning are modulated by sex hormones. Soc. Cogn. Affect. Neurosci. 7 (7), 819-830. http://dx.doi.org/10.1093/scan/nsr063.

Merz, C.J., Tabbert, K., Schweckendiek, J., Klucken, T., Vaitl, D., Stark, R., Wolf, O.T., 2012b. Oral contraceptive usage alters the effects of cortisol on implicit fear learning. Horm. Behav. 62 (4), 531-538. http://dx.doi.org/10.1016/j.yhbeh.2012.09.001.

Milad, M.R., Rauch, S.L., Pitman, R.K., Quirk, G.J., 2006. Fear extinction in rats: implications for human brain imaging and anxiety disorders. Biol. Psychol. 73 (1), 61-71. http:// dx.doi.org/10.1016/j.biopsycho.2006.01.008.

Milad, M.R., Orr, S.P., Lasko, N.B., Chang, Y., Rauch, S.L., Pitman, R.K., 2008. Presence and acquired origin of reduced recall for fear extinction in PTSD: results of a twin study. J. Psychiatr. Res. 42 (7), 515-520. http://dx.doi.org/10.1016/j.jpsychires.2008.01.017.

Ochsner, K.N., Ray, R.D., Cooper, J.C., Robertson, E.R., Chopra, S., Gabrieli, J.D.E., Gross, J.J., 2004. For better or for worse: neural systems supporting the cognitive down- and up-regulation of negative emotion. Neurolmage 23 (2), 483-499. http://dx.doi.org/ 10.1016/j.neuroimage.2004.06.030.

Ochsner, K.N., Silvers, J.A., Buhle, J.T., 2012. Functional imaging studies of emotion regulation: a synthetic review and evolving model of the cognitive control of emotion. Ann. N. Y. Acad. Sci. 1251 (1), E1-E24. http://dx.doi.org/10.1111/j.1749-6632.2012.06751. $\mathrm{X}$.

O'Connell, K., Davis, A.R., Kerns, J., 2007. Oral contraceptives: side effects and depression in adolescent girls. Contraception 75 (4), 299-304. http://dx.doi.org/10.1016/j. contraception.2006.09.008.

Oinonen, K.A., Mazmanian, D., 2002. To what extent do oral contraceptives influence mood and affect? J. Affect. Disord. 70 (3), 229-240. http://dx.doi.org/10.1016/ S0165-0327(01)00356-1.

Oinonen, K.A., Jarva, J.A., Mazmanian, D., 2008. Pre-existing hormonal differences between oral contraceptive users and nonusers? Evidence from digit ratio, age of menarche, and sociosexual orientation. In: Conti, G.A. (Ed.), Progress in Biological Psychology Research. Nova Science Publishers, Hauppauge, NY, pp. 95-116.

Österlund, M., Hurd, Y., 2001. Estrogen receptors in the human forebrain and the relation to neuropsychiatric disorders. Prog. Neurobiol. 64, 251-267. http://dx.doi.org/10. 1016/S0301-0082(00)00059-9.

Österlund, M.K., Keller, E., Hurd, Y.L., 2000. The human forebrain has discrete estrogen receptor alpha messenger RNA expression: high levels in the amygdaloid complex. Neuroscience 95 (2), 333-342. http://dx.doi.org/10.1016/S0306452299004431.

Ott, M.A., Shew, M.L., Ofner, S., Tu, W., Fortenberry, J.D., 2008. The influence of hormonal contraception on mood and sexual interest among adolescents. Arch. Sex. Behav. 37 (4), 605-613. http://dx.doi.org/10.1007/s10508-007-9302-0.

Petersen, N., Cahill, L., 2015. Amygdala reactivity to negative stimuli is influenced by oral contraceptive use. Soc. Cogn. Affect. Neurosci. 10 (9), 1266-1272. http://dx.doi.org/ $10.1093 /$ scan/nsv010.

Petersen, N., Touroutoglou, A., Andreano, J.M., Cahill, L., 2015. Oral contraceptive pill use is associated with localized decreases in cortical thickness. Hum. Brain Mapp. 36 (7), 2644-2654. http://dx.doi.org/10.1002/hbm.22797.

Pletzer, B.A., Kerschbaum, H.H., 2014. 50 years of hormonal contraception: time to find out, what it does to our brain. Front. Neurosci. 8, 256. http://dx.doi.org/10.3389/ fnins.2014.00256.

Pletzer, B., Kronbichler, M., Aichhorn, M., Bergmann, J., Ladurner, G., Kerschbaum, H.H. 2010. Menstrual cycle and hormonal contraceptive use modulate human brain structure. Brain Res. 1348, 55-62. http://dx.doi.org/10.1016/j.brainres.2010.06.019.

Pletzer, B., Kronbichler, M., Kerschbaum, H., 2015. Differential effects of androgenic and anti-androgenic progestins on fusiform and frontal gray matter volume and face recognition performance. Brain Res. 1596, 108-115. http://dx.doi.org/10.1016/j.brainres. 2014.11.025.
Raz, N., Lindenberger, U., Rodrigue, K.M., Kennedy, K.M., Head, D., Williamson, A., ... Acker, J.D., 2005. Regional brain changes in aging healthy adults: general trends, individual differences and modifiers. Cereb. Cortex 15 (11), 1676-1689. http://dx.doi.org/10. 1093/cercor/bhi044.

Rosenberg, M.J., Waugh, M.S., 1998. Oral contraceptive discontinuation: a prospective evaluation of frequency and reasons. Am. J. Obstet. Gynecol. 179 (3), 577-582. http://dx.doi.org/10.1016/S0002-9378(98)70047-X.

Sahlberg, B.L., Landgren, B.M., Axelson, M., 1987. Metabolic profiles of endogenous and ethynyl steroids in plasma and urine from women during administration of oral contraceptives. J. Steroid Biochem. 26 (5), 609-617. http://dx.doi.org/10.1016/00224731(87)90014-8.

Schmiedek, F., Lövdén, M., Lindenberger, U., 2010. Hundred days of cognitive training enhance broad cognitive abilities in adulthood: findings from the COGITO study. Front Aging Neurosci. 2, 27. http://dx.doi.org/10.3389/fnagi.2010.00027.

Shahnazi, M., Farshbaf Khalili, A., Ranjbar Kochaksaraei, F., Asghari Jafarabadi, M., Gaza Banoi, K., Nahaee, J., Bayati Payan, S., 2014. A comparison of second and third generations combined oral contraceptive pills' effect on mood. Iran. Red Crescent Med. J. 16 (8), e13628. http://dx.doi.org/10.5812/ircmj.13628.

Siegle, G.J., Konecky, R.O., Thase, M.E., Carter, C.S., 2003. Relationships between amygdala volume and activity during emotional information processing tasks in depressed and never-depressed individuals: an fMRI investigation. Ann. N. Y. Acad. Sci. 985 481-484. http://dx.doi.org/10.1111/j.1749-6632.2003.tb07105.x.

Svendal, G., Berk, M., Pasco, J.A., Jacka, F.N., Lund, A., Williams, L.J., 2012. The use of hormonal contraceptive agents and mood disorders in women. J. Affect. Disord. 140 (1), 92-96. http://dx.doi.org/10.1016/j.jad.2012.03.030.

Toffoletto, S., Lanzenberger, R., Gingnell, M., Sundström-Poromaa, I., Comasco, E., 2014 Emotional and cognitive functional imaging of estrogen and progesterone effects in the female human brain: a systematic review. Psychoneuroendocrinology 50 28-52. http://dx.doi.org/10.1016/j.psyneuen.2014.07.025.

United Nations, Department of Economic and Social Affairs, Population Division, 2015 World Contraceptive Use 2015 (POP/DB/CP/Rev2015) [Data file]. Retrieved from http://www.un.org/en/development/desa/population/publications/dataset/ contraception/wcu2015.shtml.

Van Wingen, G.A., van Broekhoven, F., Verkes, R.J., Petersson, K.M., Bäckström, T., Buitelaar, J., Fernández, G., 2007. How progesterone impairs memory for biologically salient stimuli in healthy young women. J. Neurosci. 27 (42), 11416-11423. http://dx. doi.org/10.1523/JNEUROSCI.1715-07.2007.

Van Wingen, G.A., van Broekhoven, F., Verkes, R.J., Petersson, K.M., Bäckström, T., Buitelaar, J.K., Fernández, G., 2008. Progesterone selectively increases amygdala reactivity in women. Mol. Psychiatry 13 (3), 325-333. http://dx.doi.org/10.1038/sj.mp. 4002030.

Van Wingen, G.A., Ossewaarde, L., Bäckström, T., Hermans, E.J., Fernández, G., 2011. Gonadal hormone regulation of the emotion circuitry in humans. Neuroscience 191 38-45. http://dx.doi.org/10.1016/j.neuroscience.2011.04.042.

Vandenberg, S.G., Kuse, A.R., 1978. Mental rotations, a group test of three-dimensional spatial visualization. Percept. Mot. Skills 47 (2), 599-604. http://dx.doi.org/10.2466/ pms.1978.47.2.599.

Ward, B., 2000. Simultaneous Inference for fMRI Data. Biophysics Research Institute, Medical College of Wisconsin Retrieved from http://afni.nimh.nih.gov/pub/dist/doc/ manual/AlphaSim.pdf.

Warren, A.M., Gurvich, C., Worsley, R., Kulkarni, J., 2014. A systematic review of the impact of oral contraceptives on cognition. Contraception 90 (2), 111-116. http://dx. doi.org/10.1016/j.contraception.2014.03.015.

Watson, D., Clark, L.A., Tellegen, A., 1988. Development and validation of brief measures of positive and negative affect: the PANAS scales. J. Pers. Soc. Psychol. 54 (6), 1063-1070. http://dx.doi.org/10.1037/0022-3514.54.6.1063.

Wiegratz, I., Kutschera, E., Lee, J.H., Moore, C., Mellinger, U., Winkler, U.H., Kuhl, H., 2003. Effect of four different oral contraceptives on various sex hormones and serumbinding globulins. Contraception 67 (1), 25-32. http://dx.doi.org/10.1016/S00107824(02)00436-5.

Zsido, R., 2014. Contributions of estradiol and hormonal contraceptive use to sex differences during fear extinction recall. Harv.Undergr. Res. J. 7 (2), 15-23. 